\title{
Comparison of the use of antibodies to $a$ smooth muscle actin and desmin in pulmonary lymphangioleiomyomatosis
}

\author{
T J Matthews, D Hornall, M N Sheppard
}

\begin{abstract}
To establish if antibodies to $\alpha$ smooth muscle actin (ASMA) are better than desmin as a tumour marker in pulmonary lymphangioleiomyomatosis both antisera were applied to five cases. ASMA strongly stained the muscle in all cases but desmin was negative. Five cases of cryptogenic fibrosing alveolitis showed that mature smooth muscle embedded in fibrous tissue surrounding cysts was positively stained with ASMA but the interstitial fibrous tissue was negative.

ASMA is a consistent and better marker than desmin for the detection of immature smooth muscle in pulmonary lymphangioleiomyomatosis.
\end{abstract}

(F Clin Pathol 1993;46:479-480)

Lymphangioleiomyomatosis (LAM) is a rare disease of young women which affects the lymphatics and lymph nodes of the mediastinum, retroperitoneum, and lung. The pulmonary disease is characterised by diffuse or focal proliferation of immature smooth muscle cells which surround terminal bronchioles, arterioles, venules and lymphatics and diffusely thicken the surrounding alveolar septa. Airway obstruction gives rise to characteristic cysts. ${ }^{1}$

Monoclonal antibodies to desmin are used in immunohistology as a marker for both striated and smooth muscle and the tumours derived from them, but the results are often poor for the identification of smooth muscle tumours. ${ }^{2}$ This is also true of attempts to use desmin antibodies to mark the smooth muscle cells in pulmonary LAM, though occasional cases have proved positive. ${ }^{3}$

Actin is a cytoskeletal protein necessary for muscle contraction. Monoclonal antibodies recognising a specific smooth muscle isoform ( $\alpha$ smooth muscle actin (ASMA)) are now available, allowing smooth muscle derived tumours to be differentiated from those originating in striated muscle ${ }^{4}$ and proving superior to desmin in the diagnosis of soft tissue tumours. ${ }^{5}$

We therefore applied both antibodies to cases of pulmonary LAM and cryptogenic fibrosing alveolitis (CFA) to compare the distribution and reliability of staining of the spindle cells of LAM with the muscle and fibrous tissue seen in CFA.

\section{Methods}

Five cases of pulmonary LAM were studied, all women ranging in age from 30 to 53 years. Material from four cases was obtained at open lung biopsy and the fifth was a right lung obtained at single lung transplantation. One case also had para-aortic nodal disease and bilateral renal angiomyolipomata.

Five cases of CFA included three men and two women, aged between 49 and 61 years. All were open lung biopsy specimens with end stage fibrosis, selected for the presence of prominent muscle bundles.

Five sections of normal lung from resections for carcinoma were included to document the distribution of ASMA.

A haemotoxylin and eosin stained section from each case was reviewed and the diagnosis confirmed. Sections were cut at $3 \mu \mathrm{m}$, incubated overnight at $37^{\circ} \mathrm{C}$, dewaxed and treated with $0.5 \%$ hydrogen peroxide in methanol for 10 minutes. Sections for the demonstration of desmin were incubated with trypsin for 20 minutes; sections for ASMA were not treated according to the supplier's instructions. Sections were incubated with monoclonal antibodies to desmin (Dakopatts, Denmark clone DE-R-11), undiluted for 90 minutes, or to ASMA (Dakopatts, Denmark clone 1A4) at 1 in 30 for 60 minutes, both at room temperature. Immunoreactivity was shown by the avidin-biotin peroxidase technique and the sections counterstained with Harris's haematoxylin.

\section{Results}

In all cases of pulmonary LAM the smooth muscle proliferation lining cysts were strongly positive for ASMA (fig 1). In areas of lung, apparently normal on the haematoxylin and eosin stained slides, ASMA highlighted groups of spindle cells in the interstitium. An internal control was provided by the smooth muscle of the bronchi, bronchioles, and blood vessels.

In normal lung ASMA showed positivity of the smooth muscle around small muscular arteries, airways, and of occasional single spindle cells present in the alveolar walls.

Desmin antibody was positive in the bronchiolar smooth muscle and was much weaker in the vascular smooth muscle, but the cellular proliferations around the cystic spaces were all negative.

In the case with renal angiomyolipomas and metastasis to para-aortic lymph nodes 


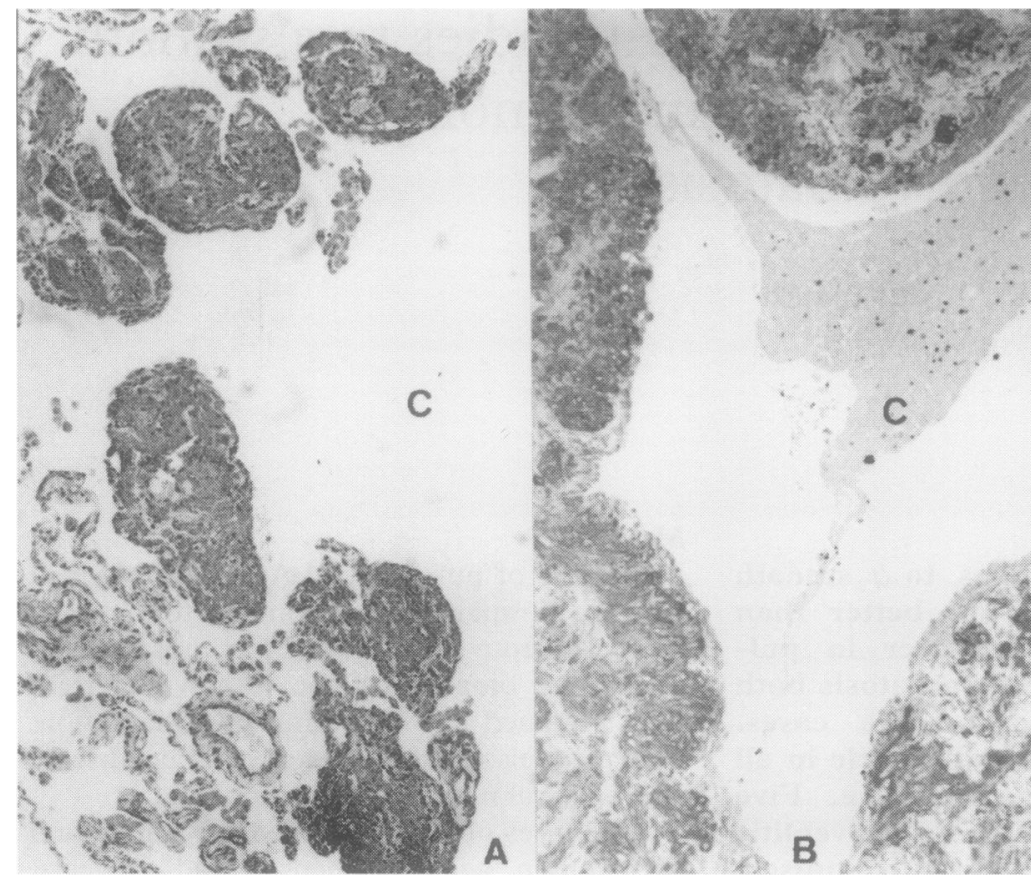

Figure 1 Cells of pulmonary LAM positive for $\alpha$ smooth muscle actin in nodular $(A)$ and diffuse (B) patterns around a cyst (C).

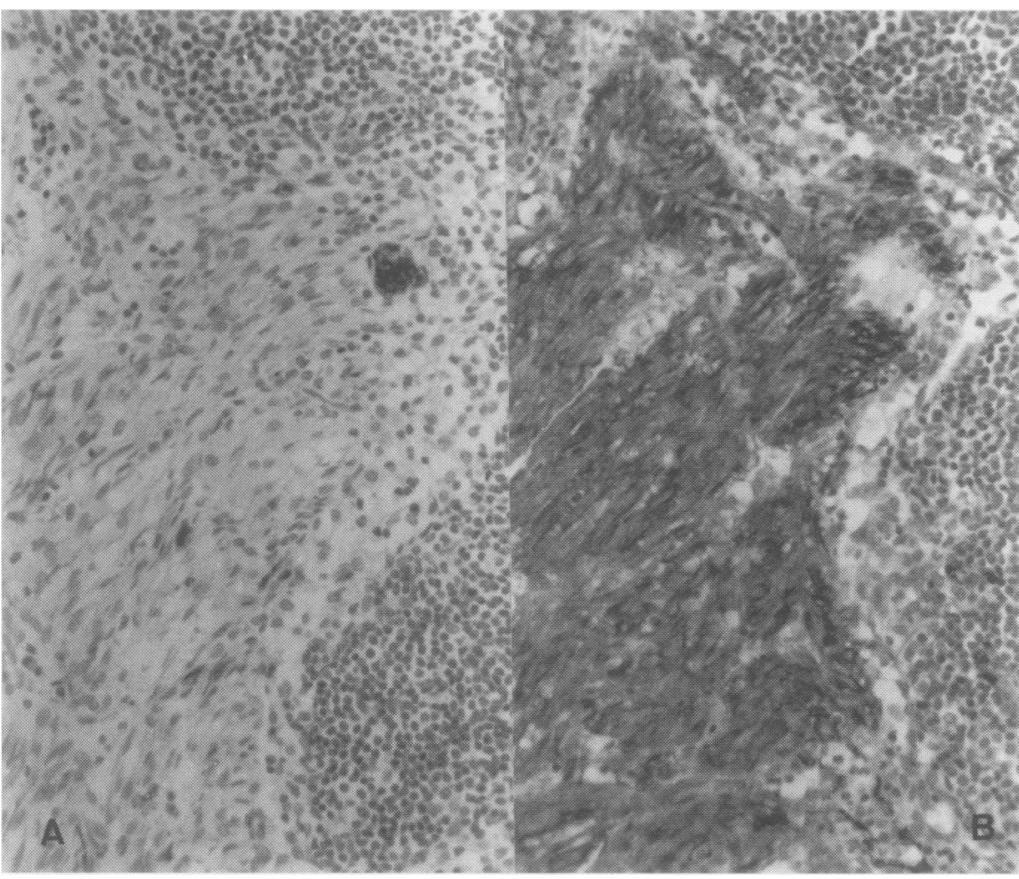

Figure 2 The same area of lymph node with desmin $(A)$ and $\alpha$ smooth muscle actin $(B)$ showing considerably more positive cells with ASMA.

both showed positivity with desmin and ASMA but with many more spindle cells positive for ASMA than desmin (fig 2).

In the cases of CFA we found positive desmin and ASMA immunoreactivity in obvious bundles of smooth muscle but the mature fibrous tissue was negative with both.

\section{Discussion}

The cellular proliferations in pulmonary LAM have been shown ultrastructurally to be smooth muscle cells ${ }^{6}$ yet most, including our five representative cases, fail to stain with desmin antibodies. This may be related to the immaturity of the smooth muscle cells, as it has been shown that the more primitive muscle tumours are rarely positive for desmin. ${ }^{7}$ It could also possibly be due to a differential distribution of desmin. In normal vascular smooth muscle muscle specific actin is diffusely present but desmin is focal and present in only half the cases. ${ }^{8}$

We achieved consistent positivity in the lesions of pulmonary LAM with ASMA, previously reported only in a single case. ${ }^{9} \mathrm{We}$ also identified clumps of positive spindle cells in apparently normal parenchyma of affected lungs which we believe are the earliest identifiable lesions of pulmonary LAM and which have not been described before.

Our findings in normal adult lung fully agree with those of Leslie $e t a l,{ }^{10}$ who suggest that the single cells in the interstitium represent pericytes of small acinar blood vessels.

In CFA we found that only recognisable smooth muscle bundles within interstitial fibrosis were positive with ASMA while the mature fibrous tissue was negative. Reactivity with ASMA has been reported in myofibroblasts identified in lung injury and repair, ${ }^{10}$ but the cases included in this study were all at a static end stage and did not include granulation tissue or areas of active fibrosis.

We conclude that ASMA is better than desmin as a marker for the proliferating smooth muscle cells characteristic of pulmonary LAM, and that it highlights an early stage that is not apparent on haematoxylin and eosin stained sections.

1 Corrin B, Liebow AA, Friedman PJ. Pulmonary lymphangiomyomatosis: A review. Am $\mathcal{F}$ Pathol 1975;79:348-67.

2 Enzinger FM, Weiss SW. Leiomyosarcoma. In: Soft tissu tumours. 2nd ed. St Louis: CV Mosby, 1988:414.

3 Buhl L, Larsen K, Bjorn-Hunsen L. Lymphangioleiomyomatosis ss fine needle aspiration cytodiagnosis possible? Acta Cytol 1988;32:559-62.

4 Bussolati G, Papotti M, Foschini MP, Eusebi V. The interest of actin immunocytochemistry in diagnostic histopathology. Basic Appl Histochem 1987;31:165-76.

5 Jones H, Steart PV, Du Boulay CE, Roche WR. Alpha smooth muscle actin as a marker for soft tissue tumours: a comparison with desmin. F Pathol 1990;162:29-33.

6 Basset F, Soler P, Marsec J, Corrin B. Pulmonary lymphangiomyomatosis. Three new cases studied with elecphangiomyomatosis. Three new cases studied

7 tron microscopy. Cancer 1976;38:2357-66. tissue tumours. 2nd edn. St Louis: CV Mosby, 1988:475. Rangdaeng S, Truong LD. Comparative immunohistochemical staining for desmin and muscle-specific actin A study of 576 cases. Am ₹ Clin Pathol 1991;96:32-45.

9 Bonetti F, Pea M, Martignoni G, Zamboni G, Iuzzolino $P$. Cellular heterogeneity in lymphangiomyomatosis of the lung. Hum Pathol 1991;22:727-8.

10 Leslie KO, Mitchell J, Woodcock-Mitchell JL, Low RB. Alpha smooth muscle actin expression in developing and adult lung. Differentiation 1990;44:143-9. 